\title{
THE CONVERGENGE OF RAYLEIGH-RITZ APPROXIMATIONS IN HYDRODYNAMICS
}

\author{
P. E. LUSH
}

\section{Introduction}

It is known that various cases of the steady isentropic irrotational motion of a compressible fluid are expressible as variational principles [1], [5]. In particular, the aerofoil problem i.e. the case of plane flow in which a uniform stream is locally deflected, without circulation, by a bounded obstacle, can be expressed in such a form. Thus we make stationary

$$
J_{1}[\phi]=\lim _{R \rightarrow \infty} \iint_{R}\left\{p-p_{\infty}+\rho_{\infty} \nabla \phi_{0} \cdot \nabla\left(\phi-\phi_{\infty}\right)\right\} d x d y
$$

where the region $R$ is that bounded internally by the obstacle $\left(C_{0}\right)$ and externally by a circle $\left(C_{R}\right)$ of radius $R$. In this expression $\phi_{\infty}$ is the velocity potential for a uniform stream, and $\phi_{0}$ is the velocity potential for the corresponding incompressible flow. It is assumed that $p$ is a function of the density $\rho$ only, and we are to express $p$ in terms of $\phi$ by use of Bernoulli's equation. The class of admissible functions is restricted to functions for which (i) $\partial \phi / \partial n=0$ on $C_{0}$, and (ii) $\phi=u_{\infty} x+v_{\infty} y+\chi$ where for $r$ large $|\chi| \leqq K^{\prime} r^{-1},|\nabla \chi| \leqq K^{\prime} r^{-2}$, the constant $K^{\prime}$ being independent of the polar angle $\theta$, and independent of the function considered.

The integral $J_{1}[\phi]$ may be used to obtain approximations to the velocity potential in the Rayleigh-Ritz manner [1]. I propose to show that in the case of a convex obstacle, if the flow is everywhere subsonic and if the approximations so obtained converge at any point $Q$ of the fluid, they converge uniformly in any bounded subregion of the fluid containing $Q$.

\section{An associated variational principle for the aerofoil problem}

In his proof of the existence of subsonic flows past a prescribed obstacle, Shiffman [2] used the integral

$$
J_{2}[\psi]=\lim _{R \rightarrow \infty} \iint_{R}\left\{p-p_{\infty}+\rho u\left(u-u_{\infty}\right)+\rho v\left(v-v_{\infty}\right)\right\} d x d y .
$$

To any $\psi$ of class $C_{2}, \rho$ is defined by Bernoulli's equation with $p=p(\rho)$, and thence $q, u, v$ by 


$$
q^{2}=u^{2}+v^{2}, \quad \rho u=\frac{\partial \psi}{\partial y}, \quad \rho v=-\frac{\partial \psi}{\partial x} .
$$

If the class of admissible functions is restricted to those (single-valued) functions for which (i) $\psi$ is constant on $C_{0}$, and (ii) $\psi=\rho_{\infty}\left(u_{\infty} y-v_{\infty} x\right)+\Psi^{\prime \prime}$ where for $r$ large $\left|\Psi^{\prime}\right| \leqq K^{\prime \prime} r^{-1},\left|\nabla \Psi^{\prime}\right| \leqq K^{\prime \prime} r^{-2}$, the constant $K^{\prime \prime}$ being independent of the angle $\theta$ and independent of the function considered. Any admissible $\psi$ which makes $J_{2}[\psi]$ stationary specifies an irrotational flow.

If we put $\psi=\rho_{\infty} \psi_{0}+\Psi$ where $\psi_{0}$ is the stream function for the corresponding incompressible flow, the last two terms of $J_{2}$ give

$$
\begin{aligned}
\lim _{R \rightarrow \infty} \iint_{R}\left\{\rho_{\infty} \frac{\partial \psi_{0}}{\partial y}\left(u-u_{\infty}\right)\right. & \left.-\rho_{\infty} \frac{\partial \psi_{0}}{\partial x}\left(v-v_{\infty}\right)-\Psi\left(u_{y}-v_{x}\right)\right\} d x d y \\
& +\lim _{\boldsymbol{R} \rightarrow \infty} \int_{\boldsymbol{C}_{0}, \boldsymbol{C}_{\boldsymbol{R}}} \Psi \boldsymbol{t} \cdot\left(\boldsymbol{q}-\boldsymbol{q}_{\infty}\right) d s .
\end{aligned}
$$

As $\Psi=O\left(r^{-1}\right), \boldsymbol{q}-\boldsymbol{q}=O\left(r^{-2}\right)$ the integral over $C_{R}$ vanishes in the limit and, as $\Psi$ is constant on $C_{0}$, the integral over $C_{0}$ vanishes for non-circulatory flow. For an extremal $u_{y}-v_{x}=0$ and as $\psi_{0}$ is conjugate to $\phi_{0}$

$$
\begin{aligned}
J_{2}\left[\psi_{\text {ext }}\right] & =\lim _{\boldsymbol{R} \rightarrow \infty} \iint_{R}\left\{p-p_{\infty}+\rho_{\infty} \nabla \phi_{0} \cdot\left(\boldsymbol{q}-\boldsymbol{q}_{\infty}\right)\right\} d x d y \\
& =J_{1}\left[\phi_{\text {ext }}\right] .
\end{aligned}
$$

For the subsonic case the extremal minimizes $J_{2}$ whereas it maximizes $J_{1}$ (Serrin [5] pp. 204-5), and it then follows that for any admissible $\phi$ and $\psi$

$$
J_{1}[\phi] \leqq J_{1}\left[\phi_{\text {ext }}\right] \leqq J_{2}[\psi]
$$

\section{Rayleigh-Ritz approximations}

For a given case of the aerofoil problem we obtain Rayleigh-Ritz approximations to the velocity potential by setting

$$
\phi_{\nu}(x, y)=\phi_{\infty}(x, y)+\sum_{i}^{\nu} A_{i} f_{i}(x, y)
$$

where, for a suitably chosen set of functions $f_{1}(x, y), f_{2}(x, y), \cdots$, we are to determine the constants $A_{i}$ so as to make $J_{1}\left[\phi_{\nu}\right]$ stationary. The functions $f_{i}$ are to be chosen so that (i) for all $\nu, \phi_{\nu}$ is an admissible function in the sense of $\S 1$; and (ii) by proper choice of the $A_{i}$, any function of the type $\chi$ defined in $\S 1$, together with its first derivatives, may be approximated to as closely as we please by $\Sigma A_{i} f_{i}$.

The (algebraic) equations for the determination of the $A_{i}$ are 


$$
\frac{\partial J_{1}}{\partial A_{i}}=\iint_{R}\left(\rho_{\infty} \nabla \phi_{0}-\rho_{\nu} \nabla \phi_{\nu}\right) \cdot \nabla f_{i} d x d y=0 \quad(i=1,2, \cdots, v)^{*}
$$

where $\rho=\rho(\phi)$. We evaluate $\phi_{\nu}$ for the values of $A_{i}$ given by these equations, and we write the equations in the equivalent form

$$
\iint_{R}\left(\rho_{\infty} \nabla \phi_{0}-\rho_{\nu} \nabla \phi_{\nu}\right) \cdot \nabla \zeta_{\nu} d x d y=0
$$

where $\zeta_{\nu}=\sum_{1}^{\nu} B_{i} f_{i}$ with $B_{i}$ arbitrary.

For any two approximations $\phi_{m}, \phi_{m+n}$ the difference $\left(\phi_{m}-\phi_{m+n}\right)$ is a function of type $\zeta-$ call it $\zeta_{m+n}$ - and by Taylor's theorem we have

$$
\begin{aligned}
J_{1}\left[\phi_{m}\right]=J_{1}\left[\phi_{m+n}\right] & +\iint_{R}\left(\rho_{\infty} \nabla \phi_{0}-\rho_{m+n} \nabla \phi_{m+n}\right) \cdot \nabla \zeta_{m+n} d x d y \\
& -\frac{1}{2} \iint_{R} \overline{\frac{\rho}{c^{2}}} Q\left[\zeta_{m+n}\right] d x d y
\end{aligned}
$$

[1]. The bar in the last term indicates that the "velocities" determining $\rho, c^{2}$ are intermediate between $\nabla \phi_{m}, \nabla \phi_{m+n}$. We use $Q$ for the quadratic expression

$$
Q[\zeta]=\left(c^{2}-u^{2}\right) \zeta_{x}^{2}-2 u v \zeta_{x} \zeta_{y}+\left(c^{2}-v^{2}\right) \zeta_{y}^{2}
$$

which, for $q^{2}=u^{2}+v^{2} \leqq q^{* 2}<c^{2}$, is positive definite in its arguments; and so for some constants $k, K$

$$
k(\nabla \zeta)^{2} \leqq \frac{1}{2} \frac{\rho}{c^{2}} Q[\zeta] \leqq K(\nabla \zeta)^{2}
$$

The second term on the right hand side of (3.3) vanishes, and if we require that the $\phi_{m}$, for all $m$, give rise to subsonic "velocities", the quadratic form $Q$ is positive definite and the $J_{1}\left[\phi_{m}\right]$ form a monotonically increasing sequence.

Since the admissible functions give rise to subsonic "velocities", we may write

$$
k_{1} \iint_{R}\left\{\nabla\left(\psi-\psi_{\infty}\right)\right\}^{2} d x d y \leqq J_{2}[\psi] \leqq K_{1} \iint_{R}\left\{\nabla\left(\psi-\psi_{\infty}\right)\right\}^{2} d x d y
$$

where $\psi_{\infty}=\rho_{\infty}\left(u_{\infty} y-v_{\infty} x\right)$ [2]. Now $\rho_{\infty} \psi_{0}$ is an admissible $\psi$, and from (2.1) it follows that

$$
J_{1}\left[\phi_{m}\right] \leqq K_{1} \iint_{R}\left\{\nabla\left(\rho_{\infty} \psi_{0}-\psi_{\infty}\right)\right\}^{2} d x d y,
$$

and thus the sequence $J_{1}\left[\phi_{m}\right]$ has a limit. Putting

$$
\left|J_{1}\left[\phi_{m}\right]-J_{1}\left[\phi_{m+n}\right]\right|<\varepsilon
$$

* We now use $R$ for the infinite region exterior to $C_{0}$. 
there follows from (3.3), (3.4)

$$
k \iint_{R}\left\{\nabla\left(\phi_{m}-\phi_{m+n}\right)\right\}^{2} d x d y<\varepsilon
$$

for $m>M(\varepsilon)$.

We use (3.5) together with a theorem due to Morrey [4] to establish the convergence of the $\phi_{m}$. Consider any two points $P, Q$ such that a semicircle upon diameter $P Q$ lies wholly within the fluid. Introduce Cartesian coordinates such that $P, Q$ are given by $( \pm a, 0)$, and let $T$ be the point $(0, a)$. Since $\nabla \phi_{m}$ is uniformly bounded

$$
\iint_{C_{r}}\left\{\nabla\left(\phi_{m}-\phi_{m+n}\right)\right\}^{2} d x d y \leqq L r^{2}
$$

for any circle $C_{r}$ of radius $r$, and Shiffman's summary [2] of Morrey's theorem then shows that

$$
\left|\zeta_{m+n}(P)-\zeta_{m+n}(Q)\right| \leqq L_{1} P Q^{\frac{1}{t}}\left\{\iint_{R}\left(\nabla \zeta_{m+n}\right)^{2} d x d y\right\}^{\frac{1}{2}}
$$

where $\zeta_{m+n}=\phi_{m}-\phi_{m+n}$ and $L_{1}$ is a constant depending on $L$.

If the semicircle $P T Q$ does not lie wholly within the fluid we may, if the surface of the obstacle is sufficiently regular, connect $P$ to $Q$ by a chain of non-overlapping semicircles lying within the fluid. For definiteness consider the case where $C_{0}$ is convex and, as it is bounded, enclose it within a square $S$. We can select a set of at most four points $P_{1}, \cdots, P_{4}$ such that, if we write $Q=P_{0}, P=P_{5}$, we can construct a set of required semicircles, one on each of the segments $P_{i-1} P_{i}, i=1, \cdots 5$. It then follows that

$$
\left|\zeta_{m+n}(P)-\zeta_{m+n}(Q)\right| \leqq L_{1}\left\{\iint_{R}\left(\nabla \zeta_{m+n}\right)^{2} d x d y\right\}^{\frac{1}{2}}\left\{Q P_{1}^{\ddagger}+\cdots+P_{4} P^{1}\right\} .
$$

Let $P$ and $Q$ be any two points of a bounded subregion $D$ of the fluid, and let $D$ be enclosed within a circle of radius $d / 2$, where $d$ is sufficiently large for the circle to enclose the square $S$, then

$$
\left|\zeta_{m+n}(P)-\zeta_{m+n}(Q)\right| \leqq 5 L_{1} d^{\ddagger}\left\{\iint_{R}\left(\nabla \zeta_{m+n}\right)^{2} d x d y\right\}^{t}
$$

From (3.5) we have finally

$$
\left|\zeta_{m+n}(P)-\zeta_{m+n}(Q)\right|<L_{2} k^{-\frac{1}{1} \varepsilon^{\ddagger}}
$$

for $m>M(\varepsilon)$.

If the approximations $\phi_{m}$ converge at $Q$

$$
\left|\zeta_{m+n}(Q)\right|<\varepsilon^{t}
$$

for $m>M^{\prime}(\varepsilon)$, and as the contants $L_{2}, k$ are independent of $P$, there follows from (3.7) the uniform convergence of the Rayleigh-Ritz approximations. For the case of the circular obstacle, all the $\phi_{m}$ are zero at $r=1, \theta=\frac{1}{2} \pi$, 
and thus the $\phi_{m}$ converge in any bounded subregion of the fluid containing the point $r=1, \theta=\frac{1}{2} \pi$.

Let $\phi$ be the velocity potential of the flow and set $\zeta_{m}=\phi_{m}-\phi$ then, since $\phi_{m}$ and $\phi$ are determined to an additive constant, we can adjust the constant so that the $\zeta_{m}$ are zero at some chosen point $Q$. We have from (3.6)

$$
\left|\zeta_{m}(P)\right|<L_{2}\left\{\iint_{R}\left(\nabla \zeta_{m}\right)^{2} d x d y\right\}^{\frac{1}{2}}
$$

and using (3.4), (3.3) successively there follows

$$
\left|\phi_{m}-\phi\right| \leqq L_{2} k^{-1}\left\{J_{1}[\phi]-J_{1}\left[\phi_{m}\right]\right\}^{\ddagger} \text {. }
$$

This makes definite the "criterion of mean error" of Lush and Cherry [1].

\section{References}

[1] Lush, P. E. and Cherry, T. M., The variational method in hydrodynamics. Quart. J. of Mech. and Appl. Math. 9 (1956), 6-21.

[2] Shiffman, M., On the existence of subsonic flows of a compressible fluid. J. Rat. Mech. and Anal. 1 (1952), 605-652.

[3] Courant, R., Dirichlet's Principle, Conformal Mapping, and Minimal Surfaces. Interscience. New York (1950).

[4] Morrey, C. B., Problems in the Calculus of Variations and Related Topics. Univ. of Calif. Publ. in Math. I (1943).

[5] Serrin, J., Mathematical Principles of Classical Fluid Dynamics. Handbuch der Physik Vol. 8, Springer (1959). 